\title{
A Hybrid Statistical and Prioritised Unequal Error Protection Scheme for IEEE 802.11n LDPC Codes
}

\author{
Tulsi Pawan Fowdur \\ Faculty of Engineering \\ Department of Electrical and Electronic Engineering, University of Mauritius \\ Reduit, Mauritius \\ p.fowdur@uom.ac.mu
}

\author{
Madhavsingh Indoonundon \\ Faculty of Engineering \\ Department of Electrical and Electronic Engineering, University of Mauritius \\ Reduit, Mauritius \\ madhavsingh.indoonundon@uom.ac.mu
}

\begin{abstract}
The combination of powerful error correcting codes such as Low Density Parity Check (LDPC) codes and Quadrature Amplitude Modulation (QAM) has been widely deployed in wireless communication standards such as the IEEE 802.11n and DVB-T2. Recently, several Unequal Error Protection schemes which exploit non-uniform degree distribution of bit nodes in irregular LDPC codes have been proposed. In parallel, schemes that exploit the inherent UEP characteristics of the QAM constellation have also been developed. In this paper, a hybrid UEP scheme is proposed for LDPC codes with QAM. The scheme uses statistical distribution of source symbols to map the systematic bits of the LDPC encoded symbols to the QAM constellation. Essentially, systematic symbols having highest probabilities of occurrence are mapped onto the low power region of the QAM constellation and those with a low probability of occurrence are mapped onto the high power region. The decrease in overall transmission power allows for an increased spacing between the QAM constellation points. Additionally, the scheme uses the distribution of the bit node degree of the LDPC code-word to map the parity bits having the highest degree onto prioritised QAM constellation points. Simulations with the IEEE 802.11n LDPC codes revealed that the proposed scheme can provide gains of up to $0.91 \mathrm{~dB}$ in Eb/No compared with other UEP schemes for a range of Bit Error Rate (BER) values.
\end{abstract}

Keywords - IEEE 802.11n, Low-Density-Parity-Check codes, Quadrature Amplitude Modulation, Unequal Error Protection

\section{INTRODUCTION}

In 1962, LDPC codes, which are a class of linear block codes, were invented by Gallagher [1]. After that, in 1996, David Mackay re-introduced LDPC codes [2] and in 1998 he came up with Non-Binary LDPC codes that outperformed LDPC codes [3]. Since LDPC codes can achieve near Shannon limit performances [4], they are considered to be one of the most powerful classes of error correcting codes developed to date. Consequently, several communications standards such as WiMax [5], DVB-T2 [6] and IEEE 802.11n [7] have adopted LDPC codes. The $802.11 \mathrm{n}$ standard combines QAM with LDPC codes and uses several code lengths ranging from 648 to 1944 , with code rates of $1 / 2,2 / 3,3 / 4$ and 5/6 [7], [8]. Recently, several papers have shown that the use of UEP can provide significant performance gains with LDPC codes and other coding schemes that are combined with QAM. An overview of UEP schemes developed for LDPC codes as well as schemes which exploit the UEP potential of QAM is given next.
The QAM constellation has an interesting characteristic that allows UEP to be performed as demonstrated by the bit-reordering scheme proposed in [9]. The authors [9] combined LTE Turbo codes with QAM and UEP was used to provide greater protection to the systematic bits. Consequently, significant performance gains were obtained [9]. In [10], the authors extended the work of [9] with joint source channel decoding for LTE Turbo codes. The same UEP principle was applied to IEEE 802.11n LDPC codes along with a modified hybrid ARQ scheme in [11]. An interesting scheme in [12] performed UEP by mapping more important bits of an image to variable nodes with higher degrees in irregular LDPC codes. After LDPC encoding the systematic bits were mapped onto a power efficient QAM constellation and the parity check bits onto a spectrally efficient 16-QAM constellation. Significant performance gains were obtained with the proposed scheme [12]. Additionally, in a non-binary LDPC coded modulation system, the authors [13] employed an UEP scheme based on the principle of 
bit reliability. At the BER of $10^{-5}$, the scheme achieved a gain between 0.1 and $0.5 \mathrm{~dB}$ [13]. Furthermore, in [14], structured rate-compatible codes with UEP were designed. The codes for the source-relay and source destination were optimized together. A significant gain was obtained over conventional LDPC codes and punctured rate compatible LDPC codes [14]. Finally, in [15], a statistical QAM based modulation scheme for low complexity video transmission was proposed. The rationale behind the scheme was to map the most frequent pixel values onto the QAM constellation points with the lowest energy. Consequently, the average energy needed for image transmission was much smaller and allowed for increasing the spacing between QAM constellation points for the same average energy thus improving the BER [15].

In this paper, a hybrid UEP scheme for binary LDPC codes with QAM is proposed. In this scheme, the source is assumed to be a set of variable-length coded characters with unequal probability distribution. The scheme then uses the statistical distribution of the source symbols to map the systematic bits of the LDPC encoded symbols to the QAM constellation. Essentially, systematic symbols having highest probabilities of occurrence are mapped onto the low power region of the QAM constellation as in [15], and those with low probabilities of occurrence are mapped onto the high power region. Consequently, the spacing between constellation points can be increased for the same average energy, hence reducing the overall power required for transmission. Additionally, the scheme uses the distribution of bit node degrees of the LDPC code to map the parity bits having the highest degrees onto prioritised QAM constellation points [12]. In general, the proposed hybrid UEP scheme combines the concepts of statistical QAM (S-QAM), prioritised constellation mapping and uneven degree distribution of bit nodes with binary LDPC codes. Simulations are performed with the IEEE 802.11n LDPC codes parity check matrices. Results showed that the proposed hybrid scheme can provide gains ranging from $0.23 \mathrm{~dB}$ to $0.91 \mathrm{~dB}$ in Eb/No for code rates of $1 / 2,2 / 3$ and $3 / 4$ compared with other UEP schemes for a range of Bit Error Rate (BER) values. The contribution of this work is a new UEP scheme which consists of hybridization of performance-enhancing schemes such as UEP and S-QAM.

This paper is structured as follows. Section 2 gives the transmitter and receiver system models with the hybrid UEP scheme. Section 3 presents simulation results and analysis. Section 4 concludes the paper.

\section{TRANSMITTER AND RECEIVER SYSTEMS FOR HYBRID SCHEME}

\subsection{TRANSMITTER}

The input data is a random alphabet source with unequiprobable probability distribution. The letters of the alphabet present the alphabet source, and their corresponding probabilities are given in Table 1. Once the alphabets have been generated, variable-length cod- ing (VLC) is performed to assign code-words, as given in Table 1, thereto.

The block diagram of the transmitter is shown in Fig.1.

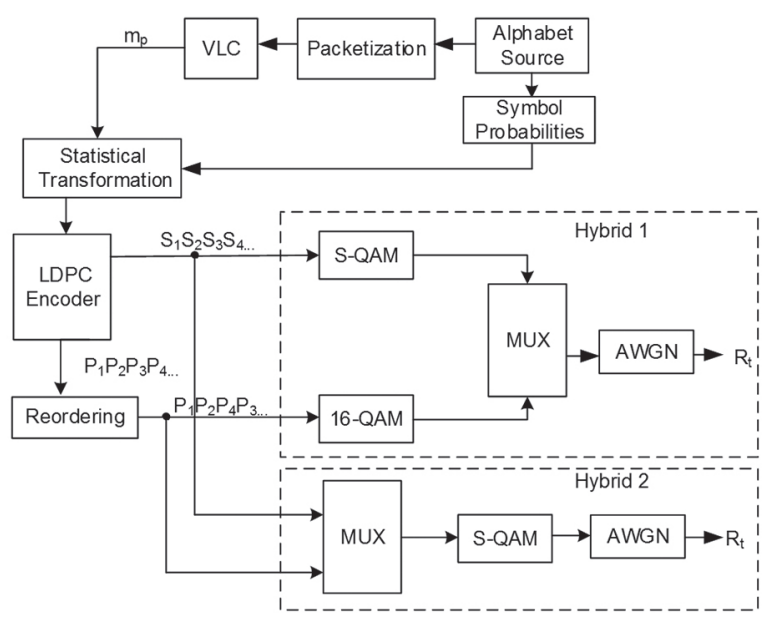

Fig. 1. Transmitter with statistical QAM transformation and bit reordering

Table 1. The alphabet source and their VLC code-words.

\begin{tabular}{|ccc|} 
Alphabet & Probability & Code-word \\
\hline a & 0.4 & 00 \\
b & 0.25 & 10 \\
\hline c & 0.2 & 11 \\
\hline d & 0.1 & 011 \\
\hline e & 0.05 & 010 \\
\hline
\end{tabular}

Let the code-word length of the LDPC code be L. If the code-rate is $\mathrm{R}$, the number of systematic bits in the code-word, $\mathrm{N}_{\mathrm{s}^{\prime}}$ is given as $\mathrm{N}_{\mathrm{s}}=\mathrm{R} \times \mathrm{L}$.

The alphabet stream is packetised by adding as many characters in the packet such that the number of bits obtained after performing variable-length coding on the characters in the packet is equal to the number of systematic bits, $\mathrm{N}_{\mathrm{s}}$. If the number of bits obtained is less than that required for LDPC encoding, redundant bits are added at the end of the packet.

In this paper, two versions of a hybrid scheme with S-QAM are proposed. The first scheme (Hybrid 1) is described now.

Statistical transformation is performed on the VLC bit stream of each packet, $m_{b}$. With S-QAM (Statistical QAM) [15], the a-priori probabilities of the QAM symbols are derived by first obtaining the a-priori probabilities of systematic bit 1 and 0 from Table 1 by using the law of total probability as follows:

$$
\begin{array}{r}
\mathrm{p}(0) \quad=p(0 \mid a) \times p(a)+p(0 \mid b) \times p(b)+p(0 \mid c) \times p(c)+ \\
p(0 \mid d) \times p(d)+p(0 \mid e) \times p(e) \\
=(1)(0.4)+(1 / 2)(0.25)+(0)(0.2) \\
+(1 / 3)(0.1)+(2 / 3)(0.05)
\end{array}
$$




$$
\begin{gathered}
p(1)=p(1 \mid a) \times p(a)+p(1 \mid b) \times p(b)+p(1 \mid c) \times p(c) \\
+p(1 \mid d) \times p(d)+p(1 \mid e) \times p(e) \\
=(0)(0.4)+(1 / 2)(0.25)+(1)(0.2) \\
+(2 / 3)(0.1)+(1 / 3)(0.05) \\
=0.4083
\end{gathered}
$$

When 16 QAM is employed $(M=16)$, the message bit stream $m_{p}$ is grouped into symbols of $4\left(\log _{2} M\right)$ bits $(0000,0001, \ldots, 1111)$. The a-priori probability of each of these symbols is given as:

$$
\text { p(symbols_(m_b ) ) }
$$

$=\prod($ probabilitity of each bit in the symbol)

For example,

$$
\mathrm{p}(0000)=\mathrm{p}(0) \times \mathrm{p}(0) \times \mathrm{p}(0) \times \mathrm{p}(0)=0.1226 .
$$

The probabilities for the whole set of symbols are given in Table 2.

After computing the probability of each message symbols, they are sent to the statistical transformation block to generate the S-QAM transformation lookup table. This block transforms the most probable symbols obtained from $\mathrm{mp}$ to the lowest power symbols and the least probable ones into the highest power symbols.

Table 2 is the statistical transformation lookup table used if 16 QAM is employed in our case:

Table 2. S-QAM transformation lookup table.

\begin{tabular}{|ccc|}
\hline Initial symbol & Probability & $\begin{array}{c}\text { Transformed } \\
\text { symbol }\end{array}$ \\
\hline 0000 & 0.1226 & 1111 \\
\hline 0001 & 0.0846 & 0001 \\
\hline 0010 & 0.0846 & 0101 \\
\hline 0011 & 0.0584 & 0011 \\
\hline 0100 & 0.0846 & 1101 \\
\hline 0101 & 0.0584 & 0100 \\
\hline 0110 & 0.0584 & 0110 \\
\hline 0111 & 0.0403 & 1110 \\
\hline 1000 & 0.0846 & 0111 \\
\hline 1001 & 0.0584 & 1001 \\
\hline 1010 & 0.0584 & 1011 \\
\hline 1011 & 0.0403 & 0000 \\
\hline 1100 & 0.0584 & 1100 \\
\hline 1101 & 0.0403 & 0010 \\
\hline 1110 & 0.0403 & 1000 \\
\hline 1111 & 0.0278 & 1010 \\
\hline
\end{tabular}

To understand the rationale behind this transformation, consider the conventional 16-QAM constellation given in Fig. 2. It is observed that 0000, which is a symbol with a high probability of occurrence, is mapped onto a high power point, whereas 1111 , which is a symbol of low probability, is mapped onto a low power point. Hence the bits in every symbol being 0000 obtained from $m_{p}$ are flipped so that these symbols become 1111. In a similar way, some bits in every symbol being 0100 (a high probability symbol) are flipped to transform the symbol to 1101 (a low power symbol). These transformations help to reduce the overall transmission power.

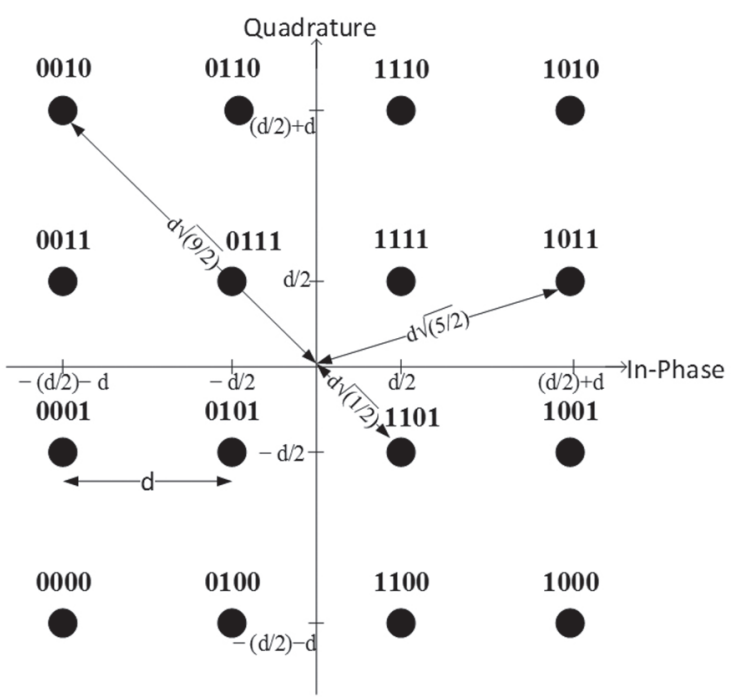

Fig. 2. 16-QAM constellation diagram.

Note that the QAM constellation point positions are not interchanged so as to preserve the UEP characteristics of the constellation. Instead, bits are flipped in a structured way in every symbol to reduce the transmission power.

LDPC encoding of the statistically transformed message obtained at the output of the Statistical Transformation block is then performed. An LDPC encoded code-word $\mathrm{c}$ for a binary message $u$ can be obtained using the generator matrix $G$ in the following matrix equation [16], [17]:

$$
\text { c=u.G ， }
$$

where,

$\mathrm{u}$ is a row vector with the message bits,

$C$ is the encoded code-word,

$\mathrm{G}$ is the generator matrix.

In this paper, the generator matrix used is as per the IEEE 802.11n LDPC codes [7].

The parity bits $\mathrm{P} 1, \mathrm{P} 2, . . \mathrm{PN}$ obtained from LDPC encoding are reordered so that they can benefit from UEP due to a prioritised mapping in the QAM constellation. The reordering is performed based on the degree distribution of parity bit nodes. The degree of a node is defined as the total number of edges linked to the node as illustrated by the Tanner graph [12]. A bit node degree is also equal to the number of $1 \mathrm{~s}$ in the column of the parity check matrix corresponding to that bit node.

A section of the Tanner graph for the IEEE $802.11 \mathrm{n}$ parity check matrix with $\mathrm{L}=648$ and rate $=1 / 2$ is given in Fig. 3 and it is clearly observed that not all bit nodes have the same degree. For example, bit nodes 350 and 351 have degree 3, while bit node 352 has degree 2 . 


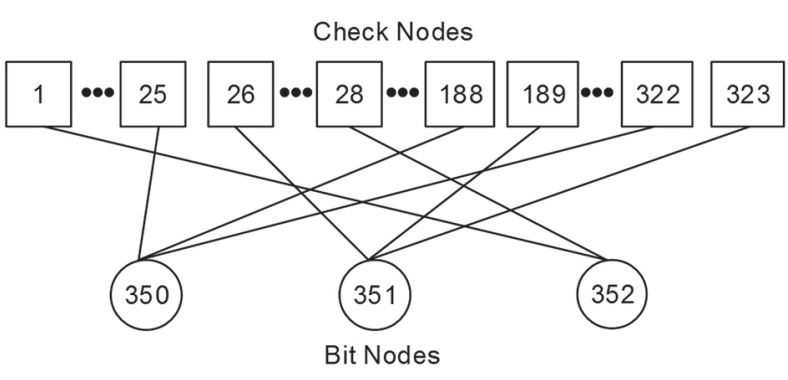

Fig. 3. Section of the Tanner graph for the $802.11 \mathrm{n}$ parity matrix with rate $1 / 2$ and code length 648 bits.

To employ UEP on parity bits, bit nodes corresponding to parity bits which have higher degrees are given more protection than those with lower degrees. For this purpose, the following reordering steps are performed on the parity bits:

1) Matrix $A_{1}$ is created, in which the degree of bit nodes corresponding to parity bits in the parity check matrix are recorded. For example, consider a section of the IEEE 802.11n LDPC parity check matrix of block length 648 with rate $1 / 2 . A$, is generated as follows:

$A_{1}=\left[\begin{array}{cccccccccccccc}\ldots & 347 & 348 & 349 & 350 & 351 & 352 & 353 & 354 & 355 & 356 & 357 & 358 & \ldots \\ \ldots & 3 & 3 & 3 & 3 & 3 & 2 & 2 & 2 & 2 & 2 & 2 & 2 & \ldots\end{array}\right]$

The first row in $A_{1}$ contains the parity bits' bit node position in the LDPC code-word and the second row contains the degrees of the bit nodes from the first row.

2) A row containing the LDPC generated parity bits is added to matrix $A_{1}$ to obtain matrix $A_{2}$. For example, if the parity bit sequence is $110110010011, A_{2}$ is obtained as follows:

$A_{2}=\left[\begin{array}{cccccccccccccc}\ldots & 347 & 348 & 349 & 350 & 351 & 352 & 353 & 354 & 355 & 356 & 357 & 358 & \ldots \\ \ldots & 3 & 3 & 3 & 3 & 3 & 2 & 2 & 2 & 2 & 2 & 2 & 2 & \ldots \\ \ldots & 1 & 1 & 0 & 1 & 1 & 0 & 0 & 1 & 0 & 0 & 1 & 1 & \ldots\end{array}\right]$

3) The columns in matrix $A_{2}$ are then sorted such that the second row is arranged in ascending order as shown below:

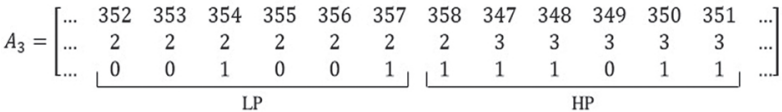

This causes the bits in the third row of $\mathrm{A}_{3}$ to be sorted in ascending order of degree and they can be extracted from the matrix.

The sorting depends only on the parity check matrix. Thus the same bit node position change will be performed on any parity bit sequence used. This process is repeated at the receiver to generate the first row of $A_{3}$ which will be used for the inverse reordering process for every transmitted packet.

In matrix $A_{3^{\prime}}$ the last 6 bits have higher degrees and thus higher priorities (HP). Therefore, they require more protection than the first 6 bits in A3 [12].

Reordering of the bits can now be performed such that the highest priority bits are given maximum protection by placing them on prioritised constellation points as explained next [10], [11].
It is observed from Fig. 2 that in each quadrant, the $1^{\text {st }}$ and $3^{\text {rd }}$ bits are the same for all four points of the quadrant. For example, in the upper right quadrant, the $1^{\text {st }}$ and $3^{\text {rd }}$ bits are 11 for all four points. Hence, the parity bits with the highest degree are placed to the $1^{\text {st }}$ and the $3^{\text {rd }}$ position. If the receiver correctly detects the quadrant of the received 16-QAM symbol, these parity bits will always be correctly detected, hence leading to improved performance.

For performing proper bit reordering in 64-QAM, a slightly modified bit mapping process is required. The sorted code-word obtained from matrix $\mathrm{A}_{3}$ is divided into 3 segments, as shown below.

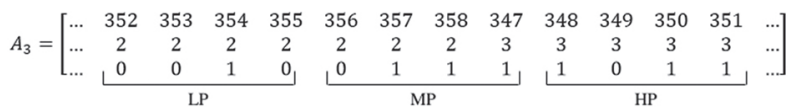

High priority (HP) bits from the sorted code-word are placed to the $1^{\text {st }}$ and the $4^{\text {th }}$ bit position in each QAM symbol. Medium priority (MP) bits from the sorted code-word are placed to the $2^{\text {nd }}$ and the $5^{\text {th }}$ bit position in each QAM symbol. Low priority (LP) bits are placed to the $3^{\text {rd }}$ and the $6^{\text {th }}$ bit position in the QAM symbols. These bit orders are based on the 64-QAM constellation of the IEEE 802.11n standard, as shown in Fig.4. This constellation has four major quadrants. In each quadrant, the $1^{\text {st }}$ and $4^{\text {th }}$ bits are the same for all 16 points. For example, in the upper right quadrant, the $1^{\text {st }}$ and $4^{\text {th }}$ bits are 11 for all 16 points. Each major quadrant is subdivided into four minor quadrants. In each minor quadrant, the $2^{\text {nd }}$ and $5^{\text {th }}$ bits are common for all four points found therein. For example, in the upper right minor quadrant, the $2^{\text {nd }}$ and $5^{\text {th }}$ bits are 00 for all four points. Hence, by placing two highest priority parity bits to the $1^{\text {st }}$ and $4^{\text {th }}$ positions, if the receiver correctly detects the major quadrant of the received symbol, these two parity bits will be correctly detected [10], [11].

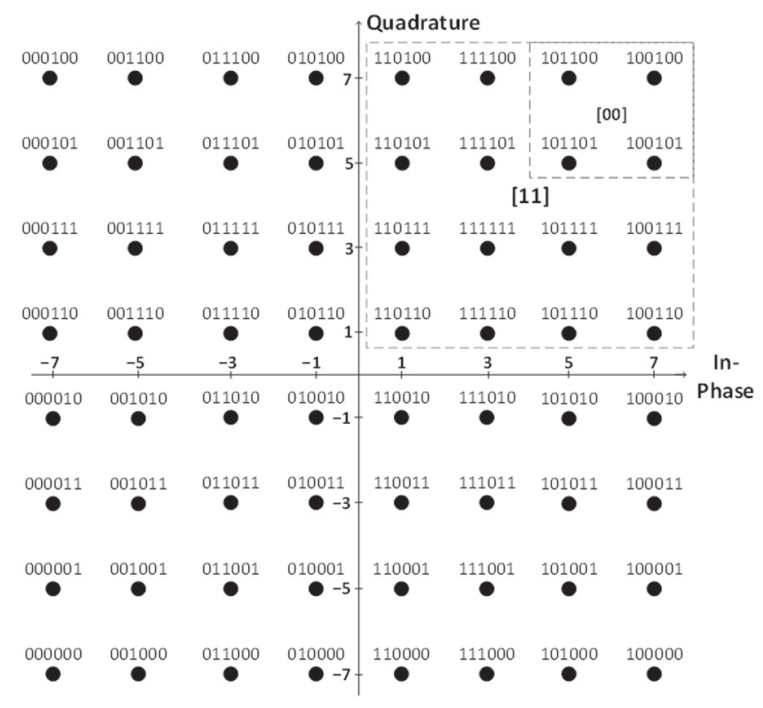

Fig. 4. The IEEE 802.11n 64-QAM constellation diagram.

After the reordering process, for the Hybrid 1 scheme, the systematic bits are modulated using S-QAM and 
the parity bits are modulated using the conventional Gray-coded QAM.

For QAM, the average power is given by:

$$
\mathrm{P}_{\mathrm{avg}}=\sum_{\mathrm{i}=0}^{M-1}\left(\mathrm{~d}_{\mathrm{i}}^{2} \times \mathrm{p}_{\mathrm{i}}\right)
$$

where,

$d_{i}$ is the distance from the symbol $i$ to the origin in the constellation diagram,

$p_{i}$ is the probability of QAM symbol $i$,

$M$ is the QAM order.

Each distance $d_{i}$ can be expressed in terms of the separation between the neighbouring symbols, $d$, as shown for a 16 QAM constellation in Fig. 2.

With the probabilities from Table $2, \mathrm{P}_{\text {avg }}$ is calculated for the standard QAM approach using the conventional constellation point separation distance $d=2\left(P_{\text {avg } 1}\right) . P_{\text {avg }}$ is then evaluated for the S-QAM approach, where more probable QAM symbols are mapped at lower power points, in terms of an unknown constellation distance $\mathrm{d}=\mathrm{ds}\left(\mathrm{P}_{\text {avg2 }}\right)$. To achieve the same power transmission for both constellations, $P_{\text {avg } 1}=P_{\text {avg2 }}$. This equation is solved to obtain ds, which will be larger than the conventional constellation point separation distance [15].

For example, using the QAM symbol probabilities from Table 2, $\mathrm{P}_{\text {avg1 }}=11.46 \mathrm{~W}$. After applying statistical transformation as in Table 2, whereby higher probability symbols are altered in order to have lower power, $P_{\text {avg }}=2.045 \mathrm{~d}_{\mathrm{s}}^{2}$. Solving $P_{\text {avg1 }}=P_{\text {avg2' }} d_{s}=2.37$. Hence for 16-QAM, $d_{s}=2.37$.

Table 3 contains the values of $d_{s}$ obtained for 16 and 64 QAM for the Hybrid 1 scheme.

Table 3. Calculated values of ds for different cases.

$\begin{array}{cc}\text { QAM order (M) } & \text { ds } \\ 16 & 2.37 \\ 64 & 2.59\end{array}$

The constellation points for the S-QAM scheme are then obtained using the value of $d_{s}$ and the increased spacing between them improves the BER performance.

For example, the constellation diagram for implementing S-QAM on LDPC codes with $M=16$ is shown in Fig.5.

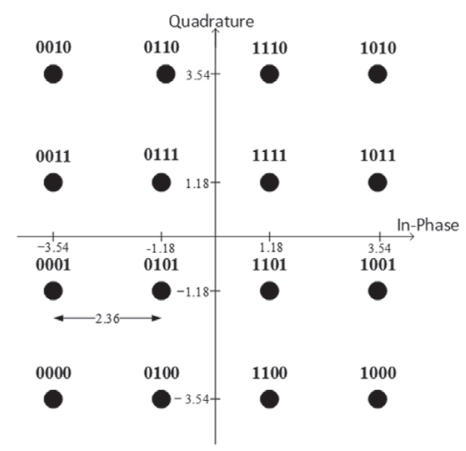

Fig. 5. The S-QAM constellation diagram for LDPC code with $M=16$.
In the Hybrid 2 scheme shown in Fig.1, the parity bits are multiplexed with systematic bits and modulated onto the S-QAM constellation instead of the conventional 16-QAM. This method allows parity bits to benefit from the increased constellation point spacing in the S-QAM constellation for improving the overall performance. Hence it is necessary to use the probability of QAM symbols obtained from the whole code-word to calculate the appropriate separation distance between the S-QAM constellation points. The a-priori probability of each QAM symbol obtained from parity bits is assumed to be equal to $1 / 16$ (equiprobable symbols). Thus the a-priori probability of each QAM symbol obtained from the whole code-word is calculated as follows:

$$
\begin{aligned}
& \mathrm{p}\left(\text { Symbol }_{\text {codeword }}\right)=\mathrm{p}\left(\text { Symbol }_{\mathrm{m}_{\mathrm{b}}}\right) \times \mathrm{R} \\
& +\mathrm{p}\left(\text { Symbol }_{\text {parity }}\right) \times(1-\mathrm{R})^{\prime}
\end{aligned}
$$

where $\mathrm{R}$ is the LDPC code-rate given by the ratio of the number of systematic bits to the total number of bits in the code-word.

For example, if $\mathrm{R}=1 / 2$,

$$
\begin{aligned}
& \mathrm{p}(0000 \text { message })=0.1226 \\
& \mathrm{p}(0000 \text { parity }) \quad=1 / 16 \\
& \mathrm{p}(0000 \text { code-word })=0.1226 \times(1 / 2) \\
& \quad+(1 / 16) \times(1-1 / 2)=0.09255
\end{aligned}
$$

The probabilities of the remaining symbols are obtained in a similar way.

Using the same method as for the Hybrid 1 scheme but with the probabilities of the QAM symbols obtained from the whole code-word, the values of $d_{s}$ are calculated for different LDPC code-rates and QAM modulation orders. Table 4 contains the values of $d_{s}$ obtained for 16 and 64 QAM with different code-rates for the Hybrid 2 scheme.

Table 4: Calculated values of $d_{s}$ for different cases using the Hybrid 2 scheme.

\begin{tabular}{|ccc|}
\hline QAM order $(\mathrm{M})$ & Code-Rate(R) & $\mathrm{ds}$ \\
\hline 16 & $1 / 2$ & 2.17 \\
16 & $2 / 3$ & 2.23 \\
\hline 16 & $3 / 4$ & 2.26 \\
\hline 64 & $1 / 2$ & 2.31 \\
64 & $2 / 3$ & 2.40 \\
64 & $3 / 4$ & 2.44 \\
\hline
\end{tabular}

\subsection{RECEIVER}

For the Hybrid 1 scheme, the received QAM symbols, $R_{t^{\prime}}$ are first de-multiplexed into systematic and parity symbols. Systematic symbols are then demodulated using the S-QAM demodulator, whereas parity symbols are demodulated using the conventional 16-QAM demodulator, as shown in Fig.6. 
For the Hybrid 2 scheme, $R_{t}$ is first demodulated using the S-QAM demodulator to obtain soft bits which are then de-multiplexed into systematic and parity parts.

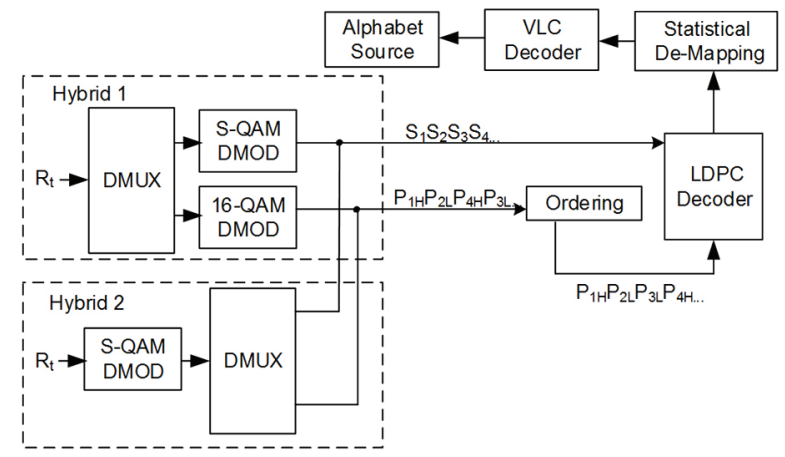

Fig. 6. Block diagram of the receiver system.

Parity soft bits are reordered back into their original positions and the systematic and reordered-back parity soft bits are sent to the LDPC decoder.

Binary LDPC decoding [1], [2], [16], depicted by the 'LDPC Decoder' block in Fig. 6, uses the Sum-Product Algorithm (SPA), which can be summarized as follows:

Step 1

These a-priori bit probabilities are expressed in terms of log-likelihood ratios (LLRs). For the AWGN channels, the LLR of the received noisy vector $y$ is approximated as follows:

$$
\operatorname{LLR}=\frac{2 y}{\sigma^{2}},
$$

where

$y$ is the received noisy vector,

$\sigma^{2}$ is the variance of the noisy vector.

\section{Step 2}

A matrix $M$ of the same size as the parity check matrix, $\mathrm{H}$, is initialized such that it contains all LLR values at all positions where there is a 1 in $\mathrm{H}$. Matrices $A$ and $B$ are obtained from matrix $\mathrm{H}$ such that $\mathrm{A}$ stores the position of each bit node connected to each check node and B stores the position of each check node connected to each bit node.

Step 3

The extrinsic message is computed as follows:

$$
\mathrm{E}_{\mathrm{j}, \mathrm{i}}=\ln \left(\frac{1+\prod_{\mathrm{i}^{\prime} \in \mathrm{B}_{\mathrm{j},} i^{\prime} \neq \mathrm{i}} \tanh \left(\mathrm{M}_{\mathrm{j}, \mathrm{i}^{\prime}} / 2\right)}{1-\prod_{\mathrm{i}^{\prime} \in \mathrm{B}_{\mathrm{j},} i^{\prime} \neq \mathrm{i}} \tanh \left(\mathrm{M}_{\mathrm{j}, \mathrm{i}^{\prime}} / 2\right)}\right),
$$

where

$M_{\mathrm{ji}}$ is the element in matrix $M$ in row $\mathrm{j}$ and column $\mathrm{i}^{\prime}$, $B j, i^{\prime}$ is the element in matrix $B$ in row $j$ and column $i^{\prime}$.

\section{Step 4}

The total LLR of the i-th bit, $L_{i}$, is the sum of the input a priori LLRs, $r_{i}$, and the LLRs from every check node connected to the bit as follows:

$$
\mathrm{L}_{\mathrm{i}}=\mathrm{r}_{\mathrm{i}}+\sum_{\mathrm{j} \in \mathrm{A}_{\mathrm{i}}} \mathrm{E}_{\mathrm{j}, \mathrm{i}},
$$

where

$E_{i, j}$ is the element in matrix $B$ in row $j$ and column $i$, $r_{i}$ is the $i^{\text {th }}$ a priori LLR.

\section{Step 5}

The code-word is finally decoded by using the sign of the total LLR of each bit (a hard decision). Positive LLRs imply a bit decoded as a 0 , while negative LLRs imply a bit decoded as a 1.

To check whether all parity-check constraints have been satisfied, the syndrome, $\mathrm{s}$, is calculated as follows:

$$
\mathrm{s}=\mathrm{Hz}^{T} \text {, }
$$

where

$\mathrm{Z}^{\top}$ is the transposed decoded LDPC code-word vector, $\mathrm{H}$ is the LDPC parity check matrix.

If the syndrome is zero, it implies that all parity check constraints are satisfied and the decoding process is stopped. Else the decoder proceeds with the following step.

\section{Step 6}

The messages received by the check nodes from the bit nodes are calculated as follows:

$$
M_{j, i}=\sum_{j^{\prime} \in A_{i}, j^{\prime} \neq j} E_{j^{\prime}, i}+r_{i},
$$

where

$M_{\mathrm{ji}}$ is the element in matrix $M$ in row $\mathrm{j}$ and column $\mathrm{i}$, $E_{j, i}$ is the element in matrix $M$ in row $j^{\prime}$ and column $i$, $r_{i}$ is the $i^{\text {th }}$ a priori LLR.

The decoder then proceeds with the next iteration which begins with the calculation of the extrinsic message in Step 3 by using the updated matrix M.

The decoded systematic bits are sent to the Statistical De-mapping block, whereby they are grouped into blocks of log2(M) consecutive bits. The inverse S-QAM transformation is then performed according to the statistical transformation lookup table (Table 2 ) so that the message bit-stream is recovered.

Finally, the message bit-stream is decoded using a VLC decoder to recover the alphabet stream.

\section{SIMULATION RESULTS AND ANALYSIS}

The performances of the following schemes with binary LDPC codes as well 16 and 64 QAM are compared:

- Scheme 1: Hybrid 1 scheme.

- $\quad$ Scheme 2: Hybrid 2 scheme.

- Scheme 3: Hybrid 1 scheme with statistical QAM mapping but without UEP. 
- Scheme 4: Hybrid 2 scheme with statistical QAM mapping but without UEP.

- $\quad$ Scheme 5: UEP with bit reordering for both systematic and parity bits and conventional QAM.

- Scheme 6: Conventional LDPC encoding and decoding without UEP.

The simulations were performed on MATLAB ${ }^{\circledR}$ using the IEEE 802.11n LDPC matrices and the simulation parameters are as follows:

- Number of decoding iterations, $\mathbf{T}=20$.

- Channel Model: Complex AWGN.

- Modulation: 16 QAM and 64 QAM.

- Code-rates: $R=1 / 2,2 / 3$ and 3/4.

- Code-length, $\mathrm{G}=648$.

In all schemes, a total of 476191 alphabets (around 1 million bits) were packetised and transmitted.

\subsection{SIMULATION RESULTS WITH 16-QAM}

The graphs of BER against Eb/No for the six schemes using code-rates $1 / 2,2 / 3$ and $3 / 4$ with $16-\mathrm{QAM}$ are shown in Figures 7-9. It is observed that both proposed hybrid schemes, i.e. Schemes 1 and 2, provide the highest Eb/No gains compared to Scheme 6 with every tested code-rates at BER values less than $10^{-2}$. The gains that the Hybrid 1 scheme (Scheme 1) gives over Scheme 6 are $0.23 \mathrm{~dB}, 0.34 \mathrm{~dB}$ and $0.68 \mathrm{~dB}$ for code-rates $1 / 2,2 / 3$ and $3 / 4$, respectively, in the range $10^{-3} \leq \mathrm{BER} \leq 10^{-5}$. In the same BER range, the gains that the Hybrid 2 scheme (Scheme 2) gives compared to Scheme 6 are $0.3 \mathrm{~dB}, 0.68 \mathrm{~dB}$ and $0.62 \mathrm{~dB}$ for coderates $1 / 2,2 / 3$ and $3 / 4$, respectively. Hence the Hybrid 2 scheme outperforms the Hybrid 1 scheme by $0.1 \mathrm{~dB}$ with the code-rate $1 / 2$ and $0.3 \mathrm{~dB}$ with the code-rate $2 / 3$, whereas when using the code-rate 3/4 Scheme 1 gives a gain of $0.06 \mathrm{~dB}$ compared to Scheme 2 . It is also observed that in the region $B E R \geq 10^{-2}$, the proposed hybrid schemes do not provide gains compared to Schemes 5 and 6.

Scheme 5 provides Eb/No gains mainly at the BER greater than $10^{-2}$ with the maximum gain of $2 \mathrm{~dB}$, obtained when using the LDPC code-rate $1 / 2$ and the minimum gain of $0.4 \mathrm{~dB}$ obtained when using the LDPC code-rate 3/4. However, at BER values lower than $10^{-3}$, the scheme provides a gain of $0.17 \mathrm{~dB}$ with the LDPC code-rate $3 / 4$ and almost no gain with the LPDC coderate $1 / 2$. The difference in gains observed in Scheme 5 when using different code-rates is mainly due to the fact that the bit nodes have more degree variations in the tested IEEE 802.11n LDPC parity check matrix with the code-rate $3 / 4$ than the one with the code-rate $1 / 2$.

Similarly, the gain in Eb/No that Scheme 1 provides compared to Scheme 3 and that Scheme 2 provides compared to Scheme 4 is dependent on the variation in parity bit node degrees. In most cases, Scheme 1 provides a gain of $0.1 \mathrm{~dB}$ compared to Scheme 3 and
Scheme 2 provides the same gain compared to Scheme 4. Hence the hybrid schemes benefit from performance gains due to both the S-QAM and UEP characteristics.

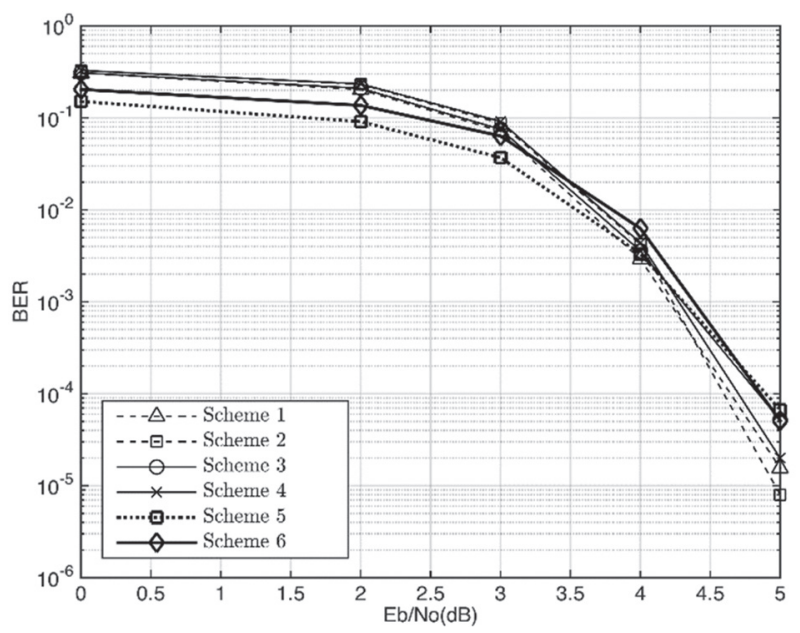

Fig. 7. Graph of Eb/No against BER using 16-QAM with $\mathrm{R}=1 / 2$.

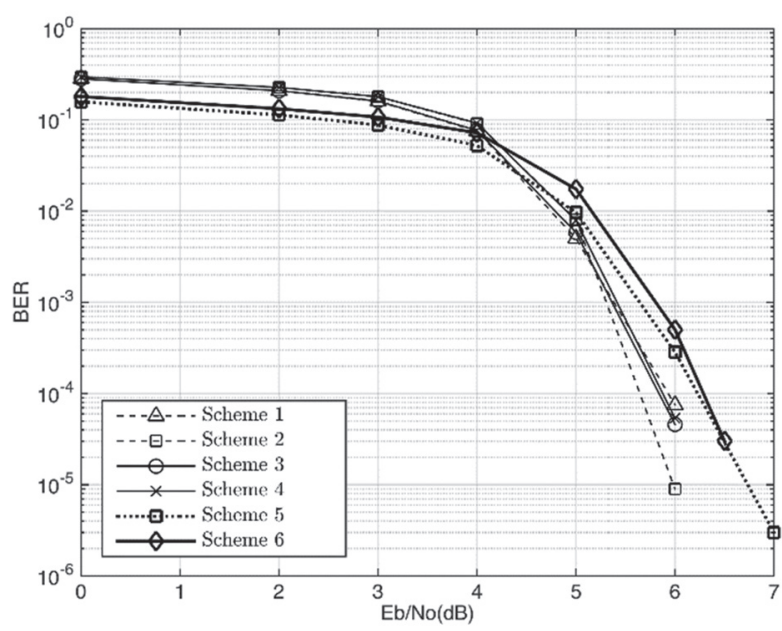

Fig. 8. Graph of Eb/No against BER using 16-QAM with $\mathrm{R}=2 / 3$.

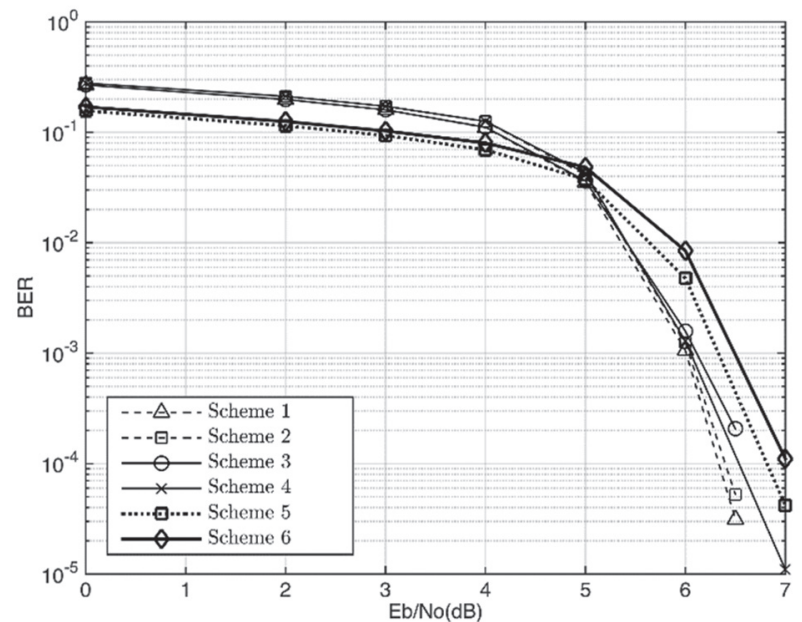

Fig. 9. Graph of Eb/No against BER using 16-QAM with $\mathrm{R}=3 / 4$. 


\subsection{SIMULATION RESULTS WITH 64-QAM}

The graphs of BER against Eb/No for the six schemes using code-rates $1 / 2,2 / 3$ and $3 / 4$ with 64-QAM are shown in Figures 10-12. It can be observed that Scheme 1 provides gains of $0.5 \mathrm{~dB}, 0.9 \mathrm{~dB}$ and 0.78 $\mathrm{dB}$ compared to Scheme 6 when using code-rates $1 / 2$, $2 / 3$ and $3 / 4$, respectively, in the range $10^{-3} \leq B E R \leq 10^{-4}$. In the same BER range, the gains that Scheme 2 gives compared to Scheme 6 are $0.3 \mathrm{~dB}, 0.46 \mathrm{~dB}$ and $0.91 \mathrm{~dB}$. Hence for 64-QAM, Scheme 1 outperforms Scheme 2 by $0.3 \mathrm{~dB}$ with the code-rate $1 / 2$ and by $0.58 \mathrm{~dB}$ except when using the code-rate $3 / 4$, whereby Scheme 2 gives a gain of $0.13 \mathrm{~dB}$ compared to Scheme 1 .

It is also observed that with 64-QAM, the Hybrid scheme does not provide a significant gain compared to the S-QAM scheme, which does not use prioritised bit ordering. This may be because with 64-QAM there are three priority levels. Moreover, the proposed hybrid schemes also do not provide gain compared to Schemes 5 and 6 in the region $B E R \geq 10^{-2}$, as observed with 16-QAM.

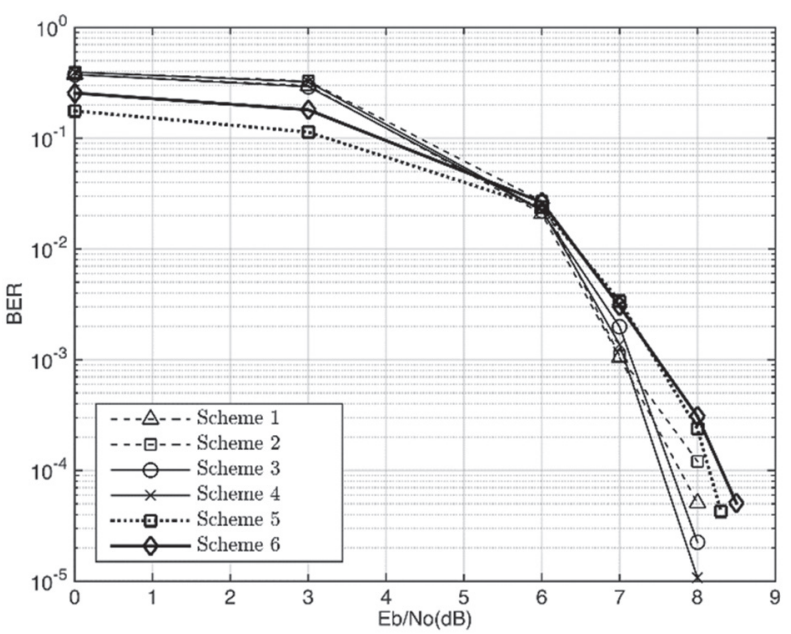

Fig.10. Graph of Eb/No against BER using 64-QAM with $R=1 / 2$.

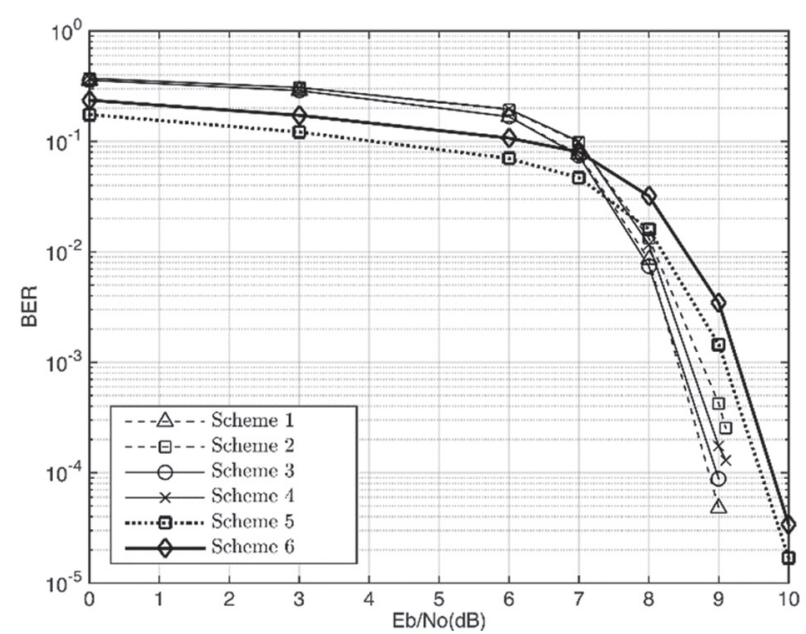

Fig.11. Graph of Eb/No against BER using 64-QAM with $\mathrm{R}=2 / 3$.

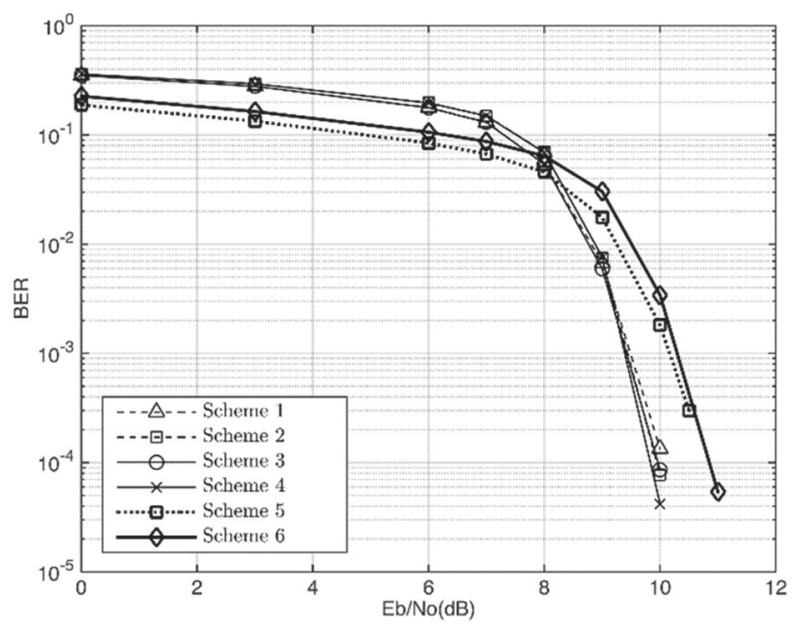

Fig.12. Graph of Eb/No against BER using 64-QAM with $\mathrm{R}=3 / 4$.

When comparing the Hybrid scheme performances with 64-QAM and with 16-QAM, it is observed that the schemes give higher gains when using 64-QAM. This can be explained by the fact that the calculated separation distances between the constellation points, $\mathrm{d}_{\mathrm{s}^{\prime}}$ for 64-QAM were larger than those calculated for 16-QAM, as shown in Table 3 and 4.

Moreover, with 16-QAM, the Hybrid 2 scheme gives higher gains than the Hybrid 1 scheme, whereas with 64-QAM, the Hybrid 1 scheme outperforms the Hybrid 2 scheme.

The proposed schemes work best with the code-rate $3 / 4$ and 2/3 LDPC codes because the bit nodes corresponding to the parity bits had more degree variations than in the code-rate 1/2 LDPC case. The performance of the proposed schemes can be improved even with the code-rate $1 / 2$ LDPC case by using an LDPC parity check matrix, whereby the bit nodes corresponding to the parity have more degree variations.

It is also clearly observed that in all simulation results obtained, both hybrid schemes proposed are outperformed by Schemes 5 and 6 in the range BER $\geq 10^{-2}$, but they effectively outperform Schemes 5 and 6 over the useful $B E R$ range, $B E R \leq 10^{-2}$.

\section{CONCLUSION}

This paper proposed two versions of a hybrid UEP scheme for the IEEE 802.11n LDPC codes. The scheme involved mapping of the QAM symbols with the highest probability occurrence onto the low power regions of the QAM constellation so as to decrease the overall transmission power, hence allowing the constellation points to be further separated so as to reduce the BER of the system. Additionally, a bit reordering scheme is incorporated prior to the QAM modulator with a view to providing better protection to the higher priority parity bits. Simulations were performed using binary LDPC codes with 16 and 64 QAM. With 16-QAM, the proposed schemes provided a maximum gain of $0.68 \mathrm{~dB}$ in $\mathrm{Eb} / \mathrm{No}$ for $\mathrm{BERs}$ lower than $10^{-2}$ and with 64-QAM, a maximum gain of $0.91 \mathrm{~dB}$ in Eb/No was 
obtained. The contribution of the paper is a new hybrid UEP scheme for LDPC codes with QAM which can provide gains of up to $0.91 \mathrm{~dB}$ in Eb/No as compared with other UEP schemes for a range of BER values. However, it was complex to tune the parameters of the hybrid schemes to obtain a gain in the $\mathrm{BER}>10^{-2}$ region and so, for future work, the proposed hybrid schemes can be further improved to obtain gains for that region. Another possible future work can be to incorporate other UEP schemes into this framework so as to obtain even further gains. Moreover, the hybrid UEP scheme can be implemented with Non-Binary LDPC codes with decoding algorithms such as the Belief-Propagation Algorithm [18], [19].

\section{REFERENCES:}

[1] R. Gallager, "Low-density parity-check codes", IRE Transactions on Information Theory, Vol. 8, No. 1, 1962, pp. 21-28.

[2] D. J. C. MacKay, R. M. Neal, “Near Shannon Limit Performance of Low Density Parity Check Codes", Electronics Letters, Vol. 33, No. 6, 1997, pp. 457-458.

[3] M. Davey, D. J. C. Mackay, "Low density parity check codes over GF(q)", IEEE Communications Letters, Vol. 2, No. 6, 1998, pp.165-167.

[4] S. Y. Chung, "On the Design of Low-Density ParityCheck Codes within $0.0045 \mathrm{~dB}$ of the Shannon Limit", IEEE Communications Letters, Vol. 5, No. 2, 2001, pp. 58-60.

[5] IEEE 802.16e Task Group, Mobile Wireless MAN, http://www.ieee802.org/16/tge/ (accessed: 2016)

[6] DVB, Digital Video Broadcasting (DVB), Implementation guidelines for a second generation digital terrestrial television broadcasting system (DVBT2), http://www.etsi.org/deliver/etsi_ts/102800_1 02899/102831/01.02.01_60/ts_102831v010201p. pdf (accessed: 2016)

[7] IEEE 802.11n-2009 Standard, http://standards. ieee.org /getieee802/download/802.11n-2009. pdf (accessed: 2016)

[8] IEEE 802.11n, "Wireless LAN Medium Access Control and Physical Layer specifications: Enhancements for Higher Throughput", IEEE P802.16n/ D1.0, March 2006.

[9] H. Lüders, A. Minwegen, P. Vary, "Improving UMTS LTE Performance by UEP in High Order Modulation", Proceedings of the $7^{\text {th }}$ International Workshop on Multi-Carrier Systems \& Solutions, Herrsching am Ammersee, Germany, 5-6 May 2009, pp.185-194.
[10] T. P. Fowdur, Y. Beeharry, K. M. S. Soyjaudah, “Performance of LTE Turbo Codes with Joint Source Channel Decoding, Adaptive Scaling and Prioritised QAM Constellation Mapping", International Journal on Advances in Telecommunications, Vol. 6, No. 3 \& 4, 2013, pp.143-152.

[11] T. P. Fowdur, B. N. Furzun, "Performance of IEEE 802.11n LDPC Codes with Modified Reliability Based Hybrid ARQ and Unequal Error Protection", Proceedings of IEEE Eurocon 2015 Conference, Salamanca, Spain, 8-11 September 2015, pp. 1-6.

[12] Y. Zhang, X. Li, H. Yang, “Unequal Error Protection in Image Transmission Based on LDPC Codes", International Journal of Signal Processing, Image Processing and Pattern Recognition, Vol. 9, No. 3, 2016, pp.1-10.

[13] L.Chen, L. Keke, F. Zesong, K. Jinming. "A novel mapping scheme in non-binary LDPC coded modulation system", Proceedings of the $11^{\text {th }}$ IEEE International Conference on Communication Technology, Hangzhou, China, 10-12 November 2008, pp. 225-228.

[14] R. Khattak, S. Sandberg, "Jointly optimized ratecompatible UEP-LDPC codes for half-duplex cooperative relay networks", EURASIP Journal on Wireless Communications and Networking, Vol. 2014, No. 1, 2014.

[15] A. Sergeev, A. Turlikov, A. Veselov, "Statistical Modulation for Low Complexity Video Transmission", Proceedings of the $11^{\text {th }}$ International Symposium on Wireless Personal Multimedia Communications, Lapland, Finland, 8-11 September 2008, pp. 33-40.

[16] S. Johnson, Introducing Low-Density Parity-Check Codes, http://materias.fi.uba.ar/6624/index_files/ outline_archivos/SJohnsonLDPCintro.pdf. (accessed: 2016).

[17] H. Kim, "Error Correction Codes", Wireless communications systems design, $1^{\text {st }}$ Ed., Wiley, 2015, p. 184.

[18] R. A. Carrasco, M. Johnston, "Non-Binary Low-Density Parity Check Codes", Non-Binary Error Control Coding for Wireless Communications and Data Storage, Wiley, 2008, pp. 208-212.

[19] C. Poulliat, M. Fossorier, D. Declercq, "Design of non binary LDPC codes using their binary image: algebraic properties", Proceedings of the 2006 IEEE International Symposium on Information Theory, Seattle, WA, USA, 9-14 July 2006, pp. 93-97. 